\title{
Intervención en Violencia de Género. Consideraciones en Torno al Tratamiento
}

\section{Intervening in Gender Violence. Considerations About the Treatment}

\author{
Inmaculada Romero \\ Dirección General de la Mujer. Comunidad de Madrid
}

\begin{abstract}
Resumen. En el momento de plantearnos una intervención psicológica con una mujer víctima de la violencia de género, se hace necesario analizar desde qué marco de valores vamos a emprender la tarea. En este trabajo consideramos una serie de aspectos que, independientemente del marco teórico de quien lleve a cabo la actuación, encuadran la intervención y le dan sentido.

Por otro lado, analizamos cuáles son los aspectos previos que deberíamos explorar en una víctima de violencia de género para no realizar intervenciones indiferenciadas, sino teniendo en cuenta la especificidad de la mujer y ayudar a su recuperación de forma más eficaz

Palabras clave: violencia, género, tratamiento, recuperación
\end{abstract}

\begin{abstract}
When considering a psychological intervention with a woman who has been a victim of gender violence, one must analyze which approach to take. Here, we weigh up several factors that ground the intervention and make it meaningful, regardless of the theoretical framework of whoever is carrying out the intervention.

We also analyze which elements of a gender violence victim's background should be considered. So, instead of performing an undifferentiated intervention, we take each woman's individuality into account and thus contribute more effectively to her recovery.

Key words: violence, gender, treatment, recovery
\end{abstract}

\section{Introducción}

A la hora de abordar las posibles intervenciones psicológicas con mujeres víctimas de violencia de género, hay que destacar, como en otros casos, la importancia de alejarse de planteamientos inespecíficos (Bleichmar,1997). Conviene tener en cuenta la particularidad de cada mujer, la fase del proceso que está atravesando, su grado de conciencia del problema, las múltiples vicisitudes que le han llevado hasta allí.

Como reto profesional es preciso, por lo tanto, diseñar modalidades de tratamientos con intervenciones especificas, adaptadas a distintas mujeres, en distintos momentos del proceso, y con distintas psicobiografías, con el fin de conseguir un alto grado de eficacia en la tarea de ayudar a las mujeres víctimas de la violencia de género en su total recuperación.

Sin embargo, y antes de esto, conviene plantear los elementos comunes y mínimos que pensamos debería cumplir toda intervención psicológica con mujeres víctimas de violencia de género, más allá de los variados modelos de intervención y más allá de las diferencias particulares entre las mujeres. Este planteamiento afectaría a cualquier intervención psicológica con mujeres

La correspondencia sobre este artículo puede dirigirse al e-mail: inmarome@yahoo.es víctimas de la violencia de género, ya sea desde un organismo público o desde el ámbito privado. Son aspectos nucleares que, a nuestro juicio, no deberían faltar en cualquier intervención, a riesgo de equivocarla.

En este trabajo pretendemos realizar un acercamiento a aquellos aspectos que consideramos que deberían estar presentes en el diseño de una intervención que afecta a la recuperación de las mujeres que han sufrido violencia, ya sea en un servicio de información y apoyo, en una casa de acogida o en un servicio especializado de atención a víctimas, y que podrían ser de utilidad para la formación de terapeutas en este ámbito.

\section{Principios básicos en la intervención}

Estos factores clave, que conviene tener en cuenta, serían los siguientes: (1) la perspectiva de género, (2) desvelar la violencia, (3) posicionamiento contra la violencia, (4) equipo multiprofesional y especializado, (5) niños y niñas: víctimas y testigos y, (6) la seguridad.

1) La perspectiva de género. La violencia contra las mujeres es una expresión del poder y dominio de los hombres sobre las mujeres, fruto de la estructura social patriarcal que asigna roles de desigual valor a hombres y mujeres, y que se traducen en determinados estereotipos de masculinidad y feminidad, con sus 
correspondientes mandatos de género, para cumplir adecuadamente lo que la sociedad patriarcal espera de unos y otras (Dio Bleichmar, 1991; Levinton, 2000). Este proceso se lleva a cabo en la primera infancia mediante la socialización diferencial entre géneros, y se va reforzando a lo largo de toda la vida por la influencia de todos los estamentos sociales y familiares impregnados a su vez de esta desigualdad de los roles entre hombres y mujeres. En este contexto generalizado de desigualdad, la mujer se convierte en una víctima probable en las relaciones familiares y esta asimetría en las relaciones se puede dejar sentir con especial intensidad en las relaciones de pareja, dando lugar entonces a la aparición de conductas de abuso basado en esa desigualdad, que paulatinamente pueden avanzar hacia una violencia cada vez más grave. Esto es así propiciado por el espacio cerrado y reducido de la intimidad de la relación, que da lugar al aislamiento y a la privacidad donde nadie puede entrar, y está reforzado por la presión del compromiso emocional y lo que ello conlleva de obligaciones, ambivalencias y lealtades. La violencia no sólo se debe a rasgos singulares y patológicos de una serie de individuos, sino que tiene rasgos estructurales de una forma cultural de definir las identidades y las relaciones entre hombres y mujeres. Estas características estructurales son las que producen que muchos individuos ejerzan la violencia contra las mujeres y, lo que es más significativo, las que permiten que la sociedad la tolere (Alberdi y Matas, 2002).

Del mismo modo, tenemos que tener en cuenta la presión que ejercen los llamados mandatos de género. En nuestra sociedad, una forma frecuente de ser mujer viene determinada por un estereotipo de feminidad tradicional que, entre otros rasgos que lo definen, incluye la atribución de una importancia fundamental de todo lo relacionado con lo emocional, con el apego y con la creación y el cuidado de las relaciones interpersonales (Dio Bleichmar, 1991), hasta el punto de que muchas mujeres adheridas a este estereotipo tienen toda su autoestima vinculada a esta tarea, y se sienten vacías y fracasadas si su relación de pareja o su familia naufragan, obligadas a aguantar cualquier cosa por no perder el apego. Podemos así entender lo que tantas veces escuchamos a estas mujeres, refiriéndose a su agresor:"¿qué voy a hacer yo sin él?", que tanta perplejidad nos causa. Por otro lado, tener en cuenta la perspectiva de género nos ayuda a poner el acento en devolver a la mujer el empoderamiento, la toma del control de su vida, que se constituye como uno de los objetivos primordiales de las intervenciones en violencia de género. Ignorar estos condicionantes de género a la hora de intervenir con las víctimas de esta violencia puede equivocar la intervención, revictimizar a la mujer y desenfocar los objetivos del trabajo. Ignorando la posición de desventaja de la mujer, se puede, por ejemplo, favorecer una labor de mediación y conciliación, que no debemos realizar en una relación donde la asimetría de poder hace imposible el diálogo. O puede desautorizar a la mujer, por considerarla inconsistente y no saber lo que quiere o, aún peor, culparle de lo que le pasa, porque de alguna manera ella lo ha elegido así.

2) Desnaturalizar la violencia invisible. Sabemos que en la sociedad y en los miembros que la componemos, la sensibilidad, el grado de conciencia, la visibilidad o el umbral de percepción (o lo que es lo mismo, la detección) de la violencia contra las mujeres es muy diverso (Gracia, 2002). Esta situación la observamos también con muchas de las mujeres maltratadas que atendemos. Hay una perspectiva subjetiva que no siempre coincide con la evaluación objetiva del trato que reciben en sus relaciones de pareja. Esta paradoja se pone claramente de manifiesto en las encuestas que se realizan para evaluar la incidencia de este problema en la sociedad. La Macroencuesta realizada por el Instituto de la Mujer en el año 2006 ha detectado un gran porcentaje de mujeres que, aún siendo claramente maltratadas (diagnosticadas como maltrato técnico), no se percibían a si mismas como maltratadas. Es decir, un gran número de mujeres están padeciendo diversos tipos de violencia "sin saberlo", considerando "normales" las conductas a las que están siendo sometidas. Es a desvelar esta "normalidad" a lo que nos referimos cuando hablamos de visibilizar y desnaturalizar el maltrato. Se trata de mujeres que viven en condiciones de desprecio, de humillación, de abuso, controladas, agredidas física o sexualmente, que viven estas situaciones con la normalidad que produce la habituación. Vemos así que gran parte de la violencia que padecen estas mujeres queda invisible para todos, incluso para quienes la padecen. Esta situación se debe a que cuando la violencia aparece en una pareja, lo hace de forma insidiosa, indetectable, con unos primeros incidentes de baja intensidad, que no pueden ser detectados como violentos por "normales" y por aislados.

Una necesidad prioritaria para las mujeres que sufren violencia consiste en desvelar todo aquello que se les ha mantenido oculto, que han considerado normal, pero que en realidad ha funcionado como peldaños de la progresión ascendente de la violencia. La reflexión crítica debe ir, precisamente, a destacar esa "anormalidad", no debemos dejar que se instauren en las relaciones entre hombres y mujeres ese tipo de conductas impositivas que en un principio parecen ser totalmente inocuas, pero que por ese modelo de relación basado en un desequilibrio de fuerzas a favor del hombre, son consideradas como territorio conquistado al que nunca se renunciará (Lorente, 2001). Es oportuno señalar que es necesario realizar esta labor de desvelamiento con las propias mujeres maltratadas para ayudarlas en su recuperación, y también con los y las profesionales que las atendemos, ya que también la subjetividad del umbral de visibilización opera en todos nosotros. 
3) Posicionamiento contra la violencia. No existe la neutralidad ante una víctima de violencia: hay que saber que hay quien ejerce violencia y hay quien la padece. La neutralidad técnica del terapeuta no es lo mismo que la neutralidad moral. Trabajar con personas victimizadas exige que se tenga una actitud moral de compromiso. Es necesario tomar una postura de solidaridad con la víctima, lo que no supone una idea simplista de que ésta no pueda hacer nada malo o equivocado, sino que "exige el entendimiento de la injusticia esencial de la experiencia traumática y la necesidad de devolver a la víctima alguna sensación de justicia", y a esto contribuye el claro posicionamiento del profesional contra la violencia (Herman, 2004, p. 214). También en palabras de Herman (2004), la neutralidad moral en el conflicto entre victima $y$ perpetrador no es una opción (...) en ocasiones, se necesita que los terapeutas elijan en qué lado están (p. 370). Sería necesario en la terapia que la mujer perciba claramente que el terapeuta está de su lado, que no está sola. Sólo desde ese lugar podemos intervenir con legitimidad para intentar apoyar a la mujer. Aquí tenemos que volver a recordar el cuestionamiento personal que deben realizar los profesionales implicados en la intervención, ya que, "un contexto terapéutico de neutralidad, secreto y privacidad no hace sino reproducir simbólicamente las condiciones en las que la violencia encuentra su mejor caldo de cultivo" (Corsi, 2003; p. 28-29).

4) Equipo multidisciplinar y especializado. Entendiendo al ser humano desde una perspectiva integral como un ser bio-psico-social, y atendiendo a la gran complejidad que presenta el fenómeno de la violencia de género, así como las múltiples necesidades que pueden presentar sus víctimas, el equipo que interviene en el proceso necesariamente deberá abarcar un amplio abanico de disciplinas que actúen de forma coordinada. Por otro lado, estos profesionales deberán estar debidamente formados en el campo de la violencia de género, dada la especificidad de esta problemática. Su formación debería abarcar varias facetas de conocimiento, como son, entre otros, los condicionantes de género y sus efectos en la salud, la subjetividad femenina, los efectos del trauma en la salud, los diagnósticos diferenciales, los fundamentos de la psicoterapia, las particularidades de este tipo de violencia y de este tipo de víctimas, etc.

Es esencial para este tipo de intervención tener en cuenta una serie de presupuestos acerca de las particularidades psicológicas que presentan estas víctimas. Es decir, no hablamos de una intervención indiferenciada o indiscriminada, sino de una intervención que debería contemplar: (a) La especificidad de estas víctimas. Muchas veces los profesionales nos encontramos con problemas para comprender a las mujeres que sufren la violencia de género (Baca y Cabanas, 2003). Las mujeres pueden tener dificultades en expresar lo que les pasa, o pueden hacerlo de forma disociada, caótica o incongruente, lo que puede hacer dudar de la veracidad de su relato. Pueden expresar una amplia gama de sentimientos de gran intensidad: "Las mujeres traumatizadas se pueden encontrar aprisionadas entre los extremos de la amnesia y de revivir el trauma, entre mareas de sentimiento intenso y abrumador y áridos estados en los que no tienen ningún sentimiento, entre la acción irritable e impulsiva y una completa inhibición de la acción" (Herman, 2004; p.85). Muchas de las mujeres maltratadas que atendemos suelen haber padecido una historia de múltiples violencias que se remonta a la infancia, y en estas situaciones de trauma prolongado y repetido, la gama de síntomas es con frecuencia mucho más compleja de la que abarca la nosología al uso. Para estas situaciones tan graves Judith Herman (2004) propone una nueva categoría diagnóstica: el Trastorno de Estrés Postraumático complejo, que da cuenta de los cambios de personalidad característicos, incluyendo las deformaciones en la capacidad de relacionarse y en la identidad. (b) La tendencia a culpar a las víctimas. Tanto por el efecto de los mandatos de género de la feminidad tradicional, que sobredimensiona lo emocional en las mujeres, y por tanto les hace "aguantar" situaciones intolerables con tal de no perder una relación de apego, como por el efecto de las traumatizaciones crónicas y repetidas, o como resultado de los múltiples obstáculos con los que se encuentran que dificulta a estas mujeres para protegerse en las relaciones íntimas, nos encontramos con mujeres que tienen grandes dificultades en terminar las relaciones con sus parejas maltratadoras, o que continúan a su lado diciendo que aún les quieren, o que inician otras relaciones con parejas abusadoras. Parecería que acepten el abuso como el precio indiscutible de una relación (Herman, 2004; p. 182). Una respuesta social frecuente consiste en culpar a las víctimas y hacerles responsables de estar padeciendo el abuso (Lorente, 1999). La tendencia a culpar a la víctima ha obstaculizado la comprensión y el diagnóstico de un síndrome postraumático; con frecuencia los profesionales de la salud mental han atribuido la situación de abuso a la supuesta psicopatología subyacente en la víctima en lugar de conceptualizar su psicopatología como una respuesta a la situación de abuso (Lorente,1999; Villavicencio y Sebastian, 1999; Corsi, 2003).

Los y las profesionales deben entender y sostener estos procesos, para evitar en la medida de lo posible la frustración y el consiguiente rechazo hacia las víctimas. Se hace entonces necesario crear un entorno terapéutico seguro y facilitador y realizar una exploración de forma especialmente cuidadosa con el fin de evitar la retraumatización, para lo que es necesario que la mujer no se sienta cuestionada, sino creída y validada. Pero no acaba aquí la especialización necesaria de los profesionales, sino que además deberán adquirir una capacitación actitudinal para hacer frente 
a la tarea de sensibilización y de cuestionamiento personal. Esto es así por las dificultades personales y profesionales derivadas de la resistencia al cambio, al tratarse de casos que implican una importante revisión de ciertas actitudes, y donde están comprometidos valores, creencias o prejuicios muy arraigados. Existen fenómenos residuales que remiten a creencias y mitos que ya se creían superados pero que se aferran a conservar su existencia y pugnan por manifestarse. En esta tarea concreta de la violencia de género, el efecto residual de esos mitos, creencias y prejuicios puede filtrarse en la escucha, el pensamiento y la intervención, operando como obstáculos en las formas de actuar (Velázquez, 2003).

Hay otros obstáculos más internos y que afectan a la propia práctica de intervención, al hecho de situarse ante una víctima de violencia. Hay que tener en cuenta el impacto que genera en la subjetividad del profesional observar e intervenir en una situación de violencia, que además se da en el marco de una relación de pareja, evocando quizá escenarios familiares propios. Es lo que llamamos las actitudes contratransferenciales, es decir, lo que pasa en el mundo interno del profesional, en su propia subjetividad en el momento del encuentro con una paciente, en este caso violentada, lo que le provoca, lo que le suscita, lo que le hace sentir. Estas actitudes contratransferenciales son a veces ignoradas o rechazadas por los profesionales. El profesional tiende a buscar la distancia óptima, entre el impacto y la reflexión. Así, su postura suele oscilar entre estas posiciones: (a) Sobreidentificación, un máximo de involucramiento personal que puede exceder las posibilidades concretas de abordar el caso, con lo que se puede aumentar la angustia del otro o (b) Rechazo, por "miedo al contagio". Debe entenderse como una forma de defensa ante la angustia que nos genera el encuentro con el problema del otro. Una de las formas sutiles del rechazo consiste en la falta de compromiso o, aún más, la neutralidad. De esta postura suele desprenderse la consideración de la víctima como culpable o, lo que es muy frecuente en estas situaciones, considerar a las víctimas como rentistas, como receptoras de beneficios secundarios. Actitud que debe entenderse como contratransferencial (Velazquez, 2003).

5) La toma en consideración de los hijos e hijas. Tanto si los niños y niñas han sufrido el maltrato físico o psicológico de forma directa como si no ha sido así, pero han sido testigos de la violencia hacia su madre, debemos considerarles como víctimas directas de esa violencia. Es decir, el estar expuestos a la violencia de género supone un daño en sí mismo, que afecta al desarrollo de los menores y debe por tanto ser considerado como maltrato (Save the Children, 2006).

Según el informe de Save the Children del año 2006, Atención a los niños y niñas víctimas de la violencia de género, "los niños y las niñas son víctimas directas en muchos casos, lo son como víctimas indirectas por- que presenciaron la violencia entre sus padres o simplemente porque viven en un entorno donde las relaciones violentas y el abuso de poder que justifica, legitima y desencadena la violencia, es parte de las relaciones afectivas y personales, internalizando un modelo negativo de relación que daña su desarrollo. Ven y sufren a una madre maltratada en vez de protectora. Ven y sufren a un padre maltratador, en vez de protector" (p. 11). Según el mismo estudio, entre los efectos de la violencia de género sobre los niños y las niñas, pueden destacarse los siguientes: (a) problemas de socialización: aislamiento, inseguridad, agresividad, (b) problemas de integración en la escuela, problemas de concentración, déficit de atención y disminución del rendimiento escolar, (c) síntomas de estrés postraumático como insomnio, fobias, ansiedad, trastornos disociativos, (d) conductas regresivas: enuresis y encopresis, (e) síntomas depresivos: llanto, tristeza y aislamiento, (f) alteraciones del desarrollo afectivo, dificultad en el manejo de emociones, aprendizaje de modelos violentos, internalización de roles de género erróneos, (g) parentalización, asumiendo roles protectores de la madre $y,(h)$ en algunos casos, la misma muerte que la madre.

Es muy importante destacar que "estas alteraciones observadas son superponibles al patrón descrito en las niñas y los niños que son víctimas directas de abusos. Por ello se empieza a incluir dentro de los tipos de maltrato infantil el hecho de estar expuesto el menor a violencia de género en el propio hogar" (Save the Children, 2006: p. 11).

Con la intervención con los menores, no sólo les ayudamos a elaborar las situaciones vividas sino que estamos realizando una fundamental tarea preventiva, minimizando el riesgo de que los comportamientos disfuncionales que les han servido de modelo se reproduzcan de generación en generación. Algunas de las conclusiones del citado informe de Save the Children son las siguientes: (a) los niños y las niñas son víctimas de la violencia de género inflingida a sus madres, y son víctimas directas en todos los casos de violencia psicológica y a veces también física, (b) existe una percepción generalizada del riesgo de que los niños y niñas repitan patrones de violencia vivida en su familia, (c) el sistema de protección a la mujer no contempla a los niños y niñas como víctimas de la violencia de género en sus acciones, lo cual posibilita su victimización secundaria por parte del sistema de protección de la mujer y, (d) el enfoque generalizado de la protección y apoyo a los niños y niñas es garantizarles la atención a través del apoyo a sus madres, no a través de medidas de apoyo psicosocial específicas y adaptadas a sus características y necesidades. En síntesis, los niños y niñas son tratados como objetos de protección y no como sujetos de derechos.

(6) La seguridad de las mujeres. De acuerdo con Herman (2004), establecer la seguridad de la mujer víctima de violencia es la primera tarea de la intervención. 
Esta tarea es prioritaria sobre todas las demás, porque ningún trabajo terapéutico puede tener éxito si no se ha establecido firmemente la seguridad. De hecho, esta autora mantiene que no se debería emprender ningún otro trabajo terapéutico hasta que se haya alcanzado un nivel razonable de seguridad. Recuperar la sensación de seguridad es un proceso que requiere un tiempo, en relación directa con la gravedad de la situación padecida. Sin embargo, este proceso puede verse obstaculizado si la mujer se encuentra con un entorno que le es hostil o que no la protege. El proceso también puede verse interrumpido con situaciones impuestas que escapan al control de la mujer, como los derivados de los procedimientos legales, en el caso de que la mujer decida denunciar la situación y/o separarse.

El primer riesgo lo supone, cómo no, la presencia del maltratador en el caso de que la mujer decida continuar con la convivencia. En el caso de que decida separarse, la violencia puede continuar en la forma de acoso o, cuando menos, estar presente como una existencia amenazante. Después, la mujer puede verse enfrentada a todo un conjunto de experiencias en distintos ámbitos (judicial, sanitario, social) en los que muchas veces vuelve a ser retraumatizada, sufriendo esta vez la victimización institucional. Todas estas circunstancias están a menudo presentes en la historia psicosocial de las mujeres y afectan directamente al proceso de recuperación.

Crear un entorno seguro para la mujer exige por tanto no sólo que se preste atención a su capacidad psicológica para protegerse a sí misma, sino también a su realidad en su entorno social. Cuidar de estos aspectos, realizar una evaluación del riesgo, apoyarle en su autoprotección, y evitar la victimización institucional, serán una garantía para su seguridad y su recuperación.

\section{Características específicas del tratamiento}

Hasta aquí hemos visto las cuestiones básicas que deberían estar presentes en el diseño de una intervención en violencia de género y, una vez analizadas, podemos pasar a considerar qué aspectos deberían tenerse en cuenta a la hora de iniciar una intervención psicológica con una mujer concreta víctima de violencia de género. Son, otra vez, aspectos cuya consideración nada tiene que ver con el enfoque teórico de quien vaya a realizar la intervención sino, más bien, cuestiones previas al inicio del tratamiento propiamente dicho. Si antes hablábamos de cuestiones tan generales que no incluían la particularidad de una mujer en concreto, sino que se referían a elementos a tener en cuenta en cualquier tratamiento de cualquier mujer maltratada, ahora justamente nos fijamos en la especificidad de la mujer para alejarnos, como señalábamos al inicio, de una intervención indiferenciada.

Es el momento de plantearnos cúal es el preciso momento de esta mujer, cómo ha codificado la historia de violencia, qué fantasías la han sostenido, hasta qué punto tiene conciencia del abuso al que ha estado sometida, cómo se ha enfrentado a él, qué recursos tiene para sobrevivir a su situación, qué precisas secuelas ha dejado la violencia, qué riesgos le acechan.

Para contestar a esas cuestiones, enfrentar la terapia con más garantías, y poder cuidar de la mujer, son varios los aspectos que nos será útil poder explorar antes de iniciar la intervención: (1) estilo de contacto, (2) grado de conciencia, (3) tipo y gravedad de la violencia y de las secuelas, (4) fase de la violencia, (5) áreas de conflicto, (6) alcance de la intervención y, (7) la seguridad de la mujer.

1) Estilo de contacto. Es interesante precisar cúal es el estilo de relación que la mujer establece con el o la profesional: puede mostrarse cercana, distante, obediente, hostil, negadora, disociada, desbordada, ya que según sea el estilo de contacto, la mujer permitirá unas $\mathrm{u}$ otras intervenciones del/a profesional (Bleichmar, 1997). Responde a los mecanismos con los que se enfrenta en general a las nuevas situaciones y que comprende, entre otras, la entrevista de exploración. Para iniciar un buen contacto psicológico, es fundamental captar rápidamente ese tono. Es importante recordar que las formas en que puede presentarse una mujer maltratada son múltiples, particulares y, en ocasiones, paradójicas. Se hace necesario conocer y comprender la especificidad de estos casos, de las que estas situaciones son alguna muestra: (a) Puede estar en shock, especialmente si la agresión está cercana en el tiempo, en una actitud de embotamiento o estupor. (b) El estado emocional que observamos puede no ser congruente con el relato de la mujer. Es relativamente habitual encontrarnos con mujeres que relatan situaciones de enorme calado emocional en un tono neutro, frívolo o incluso divertido. Se trata de un mecanismo de disociación que puede llevar al profesional a cuestionar la credibilidad de la mujer. (c) Puede estar inundada emocionalmente o manifestarse absolutamente fría. (d) Puede no tener ninguna conciencia de lo que le pasa, como hemos visto anteriormente en relación a la invisibilidad de la violencia. (e) Puede mostrar cierta hostilidad o estar a la defensiva ante nuestras intervenciones, como mecanismo de protección ante la angustia que les produce tomar conciencia de su situación. (f) Puede querer poner su vida en nuestras manos, mostrando gran pasividad y tendencia a establecer relaciones de dependencia con los profesionales, reproduciendo de esta forma otras relaciones de dependencia. Este tipo de vínculos puede suscitar en el profesional una actitud omnipotente que intente "salvar" a la mujer y que, probablemente, le generará una gran frustración posterior. (g) Puede negar la evidencia para protegerse, utilizando toda una gama de mecanismos de defensa. (h) Puede tener dificultades en expresar lo que le pasa, lo que a menudo puede malinterpretarse como dificultades intelectuales. (i) Puede manifestarse de forma disociada, caótica o incon- 
gruente, generando respuestas extremas en el profesional, haciéndole dudar de la veracidad del relato, o llevándole a realizar diagnósticos equivocados. (j) Puede presentar una historia de vida repleta de múltiples traumatizaciones, que daría lugar a una variada sintomatología, en la que la violencia actual parezca solo un episodio más. (k) Puede presentar una completa normalidad, cognitiva, afectiva y conductual, que no corresponde a lo que esperamos, y que puede hacernos dudar de su relato. Estos estados de disociación son muy frecuentes. La normalidad con que a veces asumen la violencia padecida tiene mucha importancia y suele llenar de asombro (y de rechazo) al profesional que interviene con la mujer. Esta normalidad es un fenómeno complejo que tiene distintas explicaciones, que ya hemos visto con anterioridad.

Como vemos, la variabilidad de respuestas de la mujer ante la violencia padecida puede ser muy significativa, y es importante conocerla para no hacer falsas atribuciones y también porque compromete la relación entre ella y el o la profesional. No podemos olvidar el efecto que puede producir al profesional el hecho de ser testigo del relato de la víctima. Esto hace que puedan posicionarse demasiado cerca, con el riesgo de quedar atrapados por las escenas de violencia, o demasiado lejos, con una actitud indiferente. En el espacio de la consulta, una víctima suele provocar sentimientos ambivalentes: desde franco rechazo como mecanismo defensivo frente a las manifestaciones de la violencia, hasta un máximo de involucramiento personal que puede exceder las posibilidades concretas de enfrentar el problema. Para una intervención eficaz, habrá que situarse en una distancia equilibrada entre ambas posturas.

2) Grado de conciencia. Hay una perspectiva subjetiva que no siempre coincide con la evaluación objetiva que pueda hacerse de una situación de violencia. Es decir, hay un gran número de mujeres que padecen situaciones de maltrato físico o psicológico, que no se consideran a si mismas mujeres maltratadas. Esto se debe a múltiples razones, siendo las más relevantes la naturalización de ciertas formas de violencia, que hace que se vivan como normales situaciones de abuso, y la forma insidiosa en que aparece la violencia en una relación, haciendo indetectables unos primeros incidentes por ser de baja intensidad. A esto debemos añadir los propios efectos de la violencia en las mujeres, como la habituación, la confusión o la negación como forma de protegerse. Por lo tanto, no todas las mujeres maltratadas son enteramente conscientes de ello, o lo que es lo mismo, hay un buen número de manifestaciones de violencia que no son codificadas como violentas.

Para evaluar el grado de conciencia ante una situación de abuso, y para orientar la intervención en los distintos momentos de este proceso, puede resultar útil el modelo de Fases de Cambio de Prochaska y Di Clemente. Antes de exponer los fundamentos de este modelo se debe resaltar que su utilización en este campo es limitada, ya que no es lo mismo realizar cambios en el hábito de fumar, por ejemplo, que supone una decisión individual circunscrita a un hábito particular, que realizar cambios con respecto a una relación de abuso, en la que influyen factores internos, externos y estructurales de gran complejidad, y que en muchas ocasiones se oponen a la propia dirección del cambio. Sin embargo, puede resultar de utilidad práctica para considerar el proceso de cambio y de toma de conciencia. Al trabajar con este modelo, conviene tener presente estas consideraciones para evitar simplificar la cuestión y realizar un manejo mecánico de las intervenciones.

El modelo de las Fases de Cambio propone un modelo de análisis de cómo las víctimas describen sus relaciones abusivas (Zinc, Elder, Jacobson, Klosterman, 2004). Parten de la idea de que pedir ayuda es un proceso y en él influyen distintos factores, siendo uno de ellos la identificación de una relación como abusiva. Este es un modelo presente en el ámbito sanitario para ayudar a los pacientes a realizar cambios de conducta, que busca establecer modificaciones en actitudes y comportamientos con herramientas que utiliza el propio individuo. Estas herramientas son de tipo cognitivo-afectivo y conductuales. Las fases de cambio que propone este modelo son unas fases emocionales por las que atraviesa la mujer con respecto a la toma de conciencia de su situación de violencia (calificar su relación como abusiva), y el establecimiento y mantenimiento de los cambios que realiza para acabar con esa situación (Zinc, Elder, Jacobson, Klosterman,2004). Se debe tener en cuenta que el progreso a lo largo de estas fases no es lineal, ya que pueden existir múltiples avances y retrocesos y, por otro lado, que estas fases se desarrollan en un amplio espectro de conductas, emociones, dimensiones vitales, lo que añade una enorme complejidad a un concepto como $\mathrm{el}$ cambio.

Las fases de cambio que propone este modelo son las siguientes (Zinc, Elder, Jacobson y Klostermann, 2004): (a) Fase precontemplativa. En este momento, la mujer no tiene conciencia del problema. Pueden apreciarse multitud de mecanismos defensivos que niegan, minimizan o racionalizan las conductas de abuso. Realmente, esta es una fase en la que la mujer ni tan siquiera se atreve a imaginar o sospechar que está siendo víctima de malos tratos ya que, en ese caso, esto implicaría alguna toma de decisión y, en estos momentos, no existe la menor posibilidad de que esto suceda. La mujer está sumida en un pozo sin fondo de malestar, de miedo, de confusión, sin confianza y sin conciencia clara de lo que está ocurriendo. Algunas de las intervenciones adecuadas a esta fase podrían consistir en lo siguiente: realizar un buen contacto psicológico; fomentar la duda, la interrogación acerca del abuso, de sus efectos en su salud y del riesgo; extremar la cautela en el ritmo, el cuidado en las intervenciones, ya que 
existe riesgo de abandono de la mujer; reforzar la autoeficacia, los logros mínimos; no desmontar sus mecanismos de defensa; no quitar recursos antes de haber brindado otros; tenemos que tener claro que, lo que para nosotros es un paso adelante hacia la salud, para la mujer puede constituir un paso hacia el dolor (hacia la constatación del dolor), lo desconocido, el abismo, lo incierto y, por lo tanto, lo normal es que se resista y lo evite, o que directamente se niegue o se enfrente a nuestras indicaciones; para ella, internamente, este es el momento más peligroso y doloroso, antes de atreverse siquiera a ponerle nombre a lo que le pasa; hay que tranquilizar constantemente acerca de la necesidad de abordar los pasos siguientes, nosotros los conocemos, las mujeres, no; evitar que se vaya sin dejarle la puerta abierta para otra consulta en el futuro. (b) Fase contemplativa. Por primera vez, y producto del trabajo anterior, se abre una brecha en la conciencia de la mujer, a través de la cual puede empezar a atisbar que está viviendo una situación de malos tratos y que tal vez exista alguna salida. A partir de aquí, empieza a haber algunas posibilidades de cambio, y es necesario aprovecharlas. Aquí es importante la buena intervención profesional, el acompañamiento sólido. Una vez atisbado el problema, le será más fácil encontrar estrategias para enfrentarse a ello con ayuda. De entrada, puede nombrarlo, es decir, puede decírselo a sí misma (dejar de negarlo, o negar menos, minimizar menos), y decírselo a los demás, pedir ayuda. Al poner nombre a lo que le pasa, puede empezar a reducir la confusión, puede empezar a hacer atribuciones correctas a su malestar, puede empezar a plantearse alguna toma de decisiones. Los cambios, de momento, sólo son posibilidades, nada más. Para que los cambios se produzcan, tiene que haber un análisis de los pros y los contras, y la mujer se debe sentir muy apoyada. Los cambios producen miedo, a lo que está por venir y a lo que perdemos, especialmente en estas situaciones de tanta complejidad vital. No debemos olvidar que la mujer está sólo al principio del proceso y que, por lo tanto, necesita mucho respaldo y refuerzo. (c) Fase de Preparación. La balanza se va inclinando y la mujer va realizando pequeños cambios porque ha podido empezar a ver su situación con algo más de claridad. Conviene reforzar estos pequeños cambios, porque son los que le van a proporcionar seguridad y autonomía a la mujer y los que le van a ir permitiendo dar pasos cada vez mayores. Sin embargo, suponen también un riesgo importante, ya que modifican por primera vez el equilibrio de la pareja, y el agresor puede reaccionar con violencia ante estas iniciativas de la mujer, que tiene que estar prevenida. Tanto el profesional como la propia mujer deben estar también atentos ante la ambivalencia normal en estos procesos. Los sentimientos se entremezclan y el sentido que sigue la evolución no siempre es lineal. La ambivalencia puede asustar a la mujer y hacerle creer que está equivocada en sus planteamientos o en sus sentimientos (yo no puedo sola, todavía le quiero, realmente no quiero separarme...), y al profesional puede hacerle dudar de la credibilidad de la mujer y desconfiar del proceso. (d) Fase de Acción. La mujer tiene ya una idea clara de hacia donde quiere dirigir su vida y empieza a tomar las decisiones que suponen cambios. Se trata de un reforzamiento de la fase anterior. En esta fase se toman decisiones y se realizan cambios, aumenta el riesgo y disminuye la ambivalencia. (e) Fase de Mantenimiento. La mujer tiene la fuerza suficiente para mantener los cambios que ha realizado y para haber resistido el riesgo. Ya que ha sido capaz de mantener estos logros, y que ya está situada en la vía de salida, siempre cuidando de su seguridad, es el momento de proponerle alguna intervención de tipo psicológico: terapia, grupo, grupo de autoayuda, grupos de toma de conciencia, etc. (f) Fase de Recaída. Ruptura evolutiva. En algunas ocasiones, los obstáculos han resultado insalvables para la mujer y en este momento no puede seguir adelante con la decisión de acabar con la relación de abuso. La ruptura evolutiva hace referencia a la importancia de la labor profesional en sostener el proceso, el ritmo y las decisiones de la mujer, sean cuales sean. Aunque la mujer no consiga romper con la relación en esta ocasión, son muchas las cosas que está poniendo a prueba y, si cuenta con el apoyo adecuado, le serán de gran utilidad en los futuros intentos de separación. El profesional, en esta fase, debe: ayudar a la mujer a renovar el proceso de contemplación, preparación y acción, ayudándola a que no se desmoralice; estar muy atento a los sentimientos de culpa, de autorreproche, de vergüenza, que pueden bloquear a la mujer; explicarle detenidamente el proceso de la ruptura evolutiva; estar atento a sus actitudes contratransferenciales (sentimientos de fracaso, de estafa, de ineficacia), que pueden generar hostilidad hacia la mujer; seguir positivando todo lo bueno que consiguió, a pesar de la recaída. No permitir que lo olvide; seguir confiando en ella es tal vez la única forma de que ella lo siga haciendo en sí misma y le de fuerza para la próxima ocasión.

3) Tipo y gravedad de la violencia y de las secuelas. Aunque toda violencia, incluso la de baja intensidad, produce inevitablemente un daño y, aunque los efectos son subjetivos, podemos distinguir niveles diferentes de violencia. Existe un gradiente, difícil de cuantificar, desde los más leves episodios de abuso psicológico, hasta las traumatizaciones severas y repetidas, o hasta agresiones que causan la muerte de la mujer. Todo ello irá acompañado de distintos efectos tanto en la salud física como en la salud mental (Corsi, 2003; Labrador, Rincon, De Luis, Fernandez-Velasco, 2004; Lorente, 1999; Villavicencio, 1999). No toda la violencia es igual, ni es codificada de la misma manera, ni produce el mismo daño. No se interviene igual, ni tiene las mismas necesidades, una mujer que haya sufrido leve y breve violencia psicológica en forma de desinterés, abandono emocional, falta de apoyo, que haya ido minando la confianza en sí misma, que una mujer que 
haya padecido violencia crónica desde la infancia, a manos de más de una pareja violenta e incluyendo graves agresiones sexuales. La escala del daño es continuo (aunque no necesariamente proporcional) y las intervenciones deben ser diferenciadas. El significado que adquiere la violencia para una mujer será también decisivo a la hora de evaluar el daño (Bleichmar, 1997). Es necesario evaluar las fantasías que lo sostienen: será necesario explorar cómo ha codificado las relaciones de abuso, cómo le han hecho sentirse, qué le han hecho temer, qué ha significado para ella la separación, con qué recursos cuenta para enfrentarse a todo ello.

Tendremos que evaluar las secuelas que ha producido la violencia con alguno de los cuestionarios diseñados al efecto. Así, por ejemplo, habrá que realizar una evaluación de la presencia de Trastorno de Estrés Postraumático, depresión, autoestima, ansiedad, adaptación, cogniciones postraumáticas, abuso y dependencia de sustancias (Labrador et al., 2004).

(4) Fase de la violencia. El ciclo de la violencia, descrito por la investigadora estadounidense Lenore Walker en 1984, explica cómo se produce y se mantiene la violencia en la pareja, cuáles son los sentimientos de la mujer en cada una de sus fases y, por lo tanto, ayuda a pensar cuáles pueden ser las intervenciones más adecuadas en cada una de ellas.

Tal y como lo describió, el ciclo consta de las siguientes fases (Ruiz y Blanco, 2004): (a) Fase de formación de la tensión. Se caracteriza por una escalada gradual de la tensión que se manifiesta en actos que aumentan la fricción y los conflictos en la pareja. La mujer intenta calmar la situación en la creencia irreal de que ella tiene algún control sobre la agresión. Si se le brinda ayuda, ella considera que todavía puede aguantar un poco más. Los sentimientos son angustia y miedo. (b) Fase de agresión. Estalla la violencia, del tipo que sea. La mujer denuncia la situación o habla de ella. Es el momento en que puede pedir ayuda y aceptarla. Los sentimientos de la mujer son miedo, odio, soledad, dolor e impotencia. (c) Fase de luna de miel o reconciliación. Parece que el hombre se arrepiente, hace promesas de cambio. La mujer ve "el lado bueno" de su pareja, intenta engañarse a sí misma, y hace un intento de volver a confiar en él. Esta es la fase de verdadera victimización de la mujer. Las muestras de arrepentimiento de su agresor estimulan sus sentimientos de culpa y, de acuerdo con sus mandatos de género, se ve en la obligación de no fallarle, de volver a intentar de nuevo seguir con la relación. Resulta difícil prestar ayuda a una mujer en esta fase, ya que lo vivirá como un ataque al vínculo que ella en estos momentos está luchando por preservar. Sus sentimientos son confusión, ilusión, culpa. Cuando el ciclo vuelve a comenzar, la mujer siente una enorme frustración y desilusión. Las intervenciones deben adecuarse al momento del ciclo conociendo los que serán los sentimientos preponderantes de la mujer y teniendo en cuenta lo que la mujer está en condiciones de recibir.
(5) Áreas de conflicto. Se deben explorar áreas de conflicto independientemente de si se trata de su personalidad previa o de las secuelas de la violencia. Es necesario ir analizando en qué áreas podemos iniciar la exploración y en cúales no. Para ello hay que estar muy atentos a las señales, a veces sutiles, que emite la mujer, y saber responder en consonancia. Atención a esos comentarios como "de eso prefiero no hablar", "no lo recuerdo", o señales no verbales como aumento de la actividad motora, o disminución, aparición de nerviosismo, de llanto, ausencias, etc. Habrá que valorar si se deja sin explorar, si se da un tiempo, si se da un rodeo, etc., dependiendo de la importancia que le otorgue el o la terapeuta, y teniendo siempre presente la posibilidad de retraumatizar a una víctima. Por otro lado, hay que estar atentos a síntomas o signos que pudieran hacer pensar en un cuadro psicopatológico. Conviene ir teniendo datos para hacer un diagnóstico diferencial, en el sentido de ir explorando si los síntomas que aparecen son previos o no a la violencia.

(6) Alcance de la intervención. Las mujeres maltratadas, en la complejidad de su situación psicosocial, se ven obligadas a entrar en contacto con un amplio abanico de recursos e instituciones. Algunas de las intervenciones a las que se ven sometidas pueden constituir una victimización secundaria, al no existir una buena coordinación entre las distintas instancias que acogen a la mujer. Así, se puede ver obligada a contar una y otra vez su historia y los episodios de violencia, siempre dolorosos, a profesionales distintos con distinto grado de especialización. Conviene diseñar las intervenciones a fin de que sean secuenciadas y distinguir, por ejemplo, una intervención en crisis de otro tipo de tratamiento a más largo plazo, que tendrían objetivos distintos.

(7) La seguridad de la mujer. En cualquier intervención con una mujer víctima de violencia de género, hay que tener en cuenta la seguridad de la mujer, hasta qué punto puede estar en riesgo y valorar este riesgo (Herman, 2004). "Establecer la seguridad empieza tratando el control del cuerpo y poco a poco va saliendo hacia fuera, hacia el control del entorno. Los temas de integridad física incluyen la atención a las necesidades básicas de la salud, la regulación de las funciones corporales como el sueño, la alimentación y el ejercicio, el manejo de los síntomas postraumáticos y el control de los comportamientos autodestructivos" (Herman, 2004: p. 250-251). Las cuestiones que atañen al entorno incluyen el establecimiento de una situación de vida estable, la seguridad económica, la movilidad y un plan de auto protección que incluya todo el ámbito de la vida de la mujer.

La intervención con una mujer víctima de violencia de género debe contemplar todos estos aspectos, realizando una completa exploración de los mismos, ya que pueden convertirse en obstáculos que se oponen al sentido de la recuperación. "La tarea de establecer la seguridad es especialmente compleja cuando la paciente sigue manteniendo una relación que ha sido abusiva. 
El potencial de violencia debe ser tenido en cuenta siempre, aunque la mujer diga que no tiene miedo" (Herman, 2004: p. 261). Esto es especialmente importante dado que, como consecuencia del abuso, la mujer puede presentar fallos en su autoprotección en las relaciones íntimas, que la hagan estar expuesta a otras experiencias de violencia y no detectarlo a tiempo ni poder defenderse de ello.

Otra consideración que es preciso hacer se refiere a la seguridad de la mujer en la propia terapia. Como sugiere Herman (2004: p. 266), "aunque el error terapéutico más frecuente es la evitación del material traumático en terapia, el segundo error más frecuente tal vez sea involucrarse de forma prematura o precipitada en el trabajo de exploración sin haber prestado suficiente atención a las tareas de establecer seguridad y crear una alianza terapéutica". En el mismo sentido, puede cometerse el error de abordar aspectos emocionales con la mujer para los que aún no está debidamente preparada y, tal y como sugiere Hirigoyen (1999: p. 166-167) "cuando uno empieza una psicoterapia en un contexto de acoso, no debe intentar saber primero por qué ha caído en esa situación. Lo primero es liberarse de ella inmediatamente. La psicoterapia, al menos al principio, debería resultar reconfortante y tiene que permitirle a la víctima liberarse del miedo y de la culpabilidad. Más adelante, cuando el sufrimiento disminuya y la mujer esté más restablecida, se podrá intentar comprender por qué entró en ese tipo de relación destructiva, y por qué no pudo defenderse".

\section{Conclusiones}

En este trabajo, hemos presentado algunas de las consideraciones que deben realizarse antes de iniciar el tratamiento de una mujer víctima de la violencia de género, independientemente del marco teórico del que se parta. Hemos señalado aspectos referidos, por un lado, a la posición desde la que uno, como terapeuta, se sitúa. Aspectos que afectan a la conceptualización misma de la violencia como un hecho invisible y producto de la desigualdad entre mujeres y hombres, y que atañen al compromiso del terapeuta con la víctima: su posición de compromiso, la importancia de la seguridad de las mujeres, el daño que sufren sus hijos e hijas.

Por otro lado, se han señalado aspectos referidos a la especificidad que requiere la intervención con una víctima de maltrato: la importancia que tiene su forma de vincularse, el momento de su proceso personal, el tipo y gravedad de sus secuelas y, sobre todo, el cuidado necesario que debe mantenerse para evitar su retraumatización, y evitar que vuelva a sentirse dañada y cuestionada.

La consideración de estos aspectos, realizada pre- viamente al inicio del tratamiento, puede dotar al mismo de una mayor garantía de calidad, asegurando que se tienen en cuenta aspectos clave en el encuentro con una mujer víctima de la violencia de género.

\section{Referencias}

Alberdi, I. y Matas, N. (2002). La violencia doméstica. Informe sobre los malos tratos a mujeres en España. Barcelona: Fundación La Caixa.

Baca, E. y Cabanas, M. L. (2003). Las víctimas de la violencia. Estudios psicopatológicos. Madird: Triacastela.

Bleichmar, H. (1997). Avances en psicoterapia psicoanalítica. Barcelona: Paidós.

Corsi, J. (2003). Maltrato y abuso en el ámbito doméstico. Barcelona: Paidós.

Dio Bleichmar, E. (1991). La depresión en la mujer. Madrid: Temas de Hoy.

Gracia, E. (2002). Visibilidad y tolerancia social de la violencia familiar. Intervención Psicosocial, 11, 5-15.

Herman, J. (2004). Trauma y recuperación. Madrid: Espasa Hoy.

Hirigoyen, M. F. (1999). El acoso moral. Barcelona: Paidós.

Instituto de la Mujer (2006). La violencia contra las mujeres. Resultados de la Macroencuesta. Madrid: Ministerio de Trabajo y Asuntos Sociales.

Labrador, F. J., Rincon, P. P., De Luis, P., FernandezVelasco, R. (2004). Mujeres victimas de la violencia domestica. Programa de actuación (2004). Madrid: Ediciones Pirámide.

Levinton, N. (2000). El superyó femenino. Madrid: Biblioteca Nueva.

Lorente, M. (1999). Agresión a la mujer. Maltrato, violación y acoso. Comares.

Lorente, M. (2001). Mi marido me pega lo normal. Barcelona: Crítica. Ares y Mares.

Prochask, J., Di Clemente C., Norcross J. (1994). Cómo cambia la gente. Aplicaciones en comportamientos adictivos. Revista de Toxicomanías, 1, 1-12.

Ruiz, C. y Blanco, P. (2004). La violencia en la pareja. Madrid: Díaz de Santos.

Save the Children (2006). Atención a los niños y niñas víctimas de la violencia de género en la Comunidad de Madrid.

Velázquez, S. (2003). Violencias cotidianas, violencia de género. Buenos Aires: Paidós.

Villavicencio, P. y Sebastian, J. (1999). Violencia doméstica; su impacto en la salud física y mental de las mujeres. Madrid: Instituto de la mujer.

Walker, L. (1984). The Battered woman Syndrome. Springer. New York.

Zinc T., Elder N., Jacobson J., Klostermann, B. (2004). Medical management of intimate partner violence considering the stages of change: precontemplation and contemplation. Annals of Family Medicine, 2, 231-239.

Manuscrito Recibido: 15/09/2009

Revisión Recibida: 25/01/2010

Manuscrito Aceptado: 25/01/2010 